\title{
HIPERTENSÃO PULMONAR ESQUISTOSSOMÓTICA PERSISTENTE EM PACIENTE APÓS REVERSÃO DA FORMA HÉPATO-ESPLÊNICA: APRESENTAÇÃO DE UM CASO
}

\author{
Antônio Emanuel1, Aluizio Prata1, J.C. Bina2 e Armênio Guimarães² \\ Os autores relatam o caso de um paciente masculino, de 24 anos, hépato-esplênico \\ e com hipertensão pulmonar esquistossomótica (pressão média na artéria pulmonar de \\ 27,5mm HG). Tratado com oxamniquine. Após 11 anos o exame mostrou reversão à \\ hépato-intestinal, com persistência da hipertensào pulmonar, diagnosticada pelo cate- \\ terismo cardíaco (pressão média na artéria pulmonar de $20 \mathrm{~mm} \mathrm{Hg}$ ) e ecocardiografía.
}

Palavras chaves: Esquistossomose. Hipertensão pulmonar. Hipertensão porta.

Depois que Azmy ${ }^{1}$ assinalou o primeiro caso de hipertensão pulmonar esquistossomótica, outros relatos vieram a ser feitos, e todos os pacientes tinham a forma hépato-esplênica da esquistossomose 67111218 . Para Coelho e Duarte ${ }^{5}$, Kenawy ${ }^{10}$ e Ibrahim e Girgis ${ }^{9}$ o diagnóstico de hipertensão pulmonar esquistossomótica não poderia ser feito na ausência de hépato-esplenomegalia. Barbato ${ }^{2}$ menciona a existencia de hipertensão pulmonar esquistossomótica na ausêricia de hépato-esplenomegalia. Cavalcanti e cols ${ }^{4}$ referem que em 30 pacientes diagnosticados inclusive pela hemodinâmica, 10 não eram hépato-esplênicos. Não hà outras referências de hipertensão pulmonar em pacientes sem a forma hépato-esplênica, embora Santiago e Ratton ${ }^{15}$ mencionem que ela possa existir "excepcionalmente nos portadores da forma intestinal".

Neste trabalho apresentaremos um paciente com hipertensão pulmonar, diagnosticada pelo cateterismo cardíaco, no qual houve reversão da forma hépato-esplênica.

\section{APRESENTAÇÃO DO CASO}

A.S.T., 24 anos, masculino, branco solteiro, lavrador, natural e procedente de Taquarandi-BA.Em 1972 apresentava figado a $2 \mathrm{~cm}$ da reborda costal direita e a $7 \mathrm{~cm}$ do apêndice xifóide, e baço a $3 \mathrm{~cm}$ da reborda costal esquerda. Foi cateterizado e a pressão média na artéria pulmonar foi de $27,5 \mathrm{mmHg}$. Foi considerado como hépato-esplênico e tendo hipertensão pulmonar esquistossomótica. Em 03/02/83 foi examinado clinicamente, queixava-se de dor abdominal, tipo cólica, de moderada intensidade, difusa, periódica e diarréia com 4-5 dejeções ao dia, muco,

1. Núcleo de Medicina Tropical e Nutrição da Universidade de Brasília, 70910 Brasilia.

2. Faculdade de Medicina da Universidade Federal da Bahia.

Recebido para publicação em 19/12/85. sangue e tenesmo. Não tinha dispnéia, dor precordial, escurecimento visual, tonturas ou sincopes. Nunca teve hematêmese ou melena. Relatava já ter feito tratamento especifico para esquistossomose após o exame de 1972. Ao exame apresentava pulso de 64 batimentos/minuto, ritmico. TA-120/70 $\mathrm{mmHg}$, mucosas-coradas. $\mathrm{O}$ tórax tinha conformação normal e o exame do aparelho respiratório não apresentou anormalidades. $O$ ictus foi palpado em decúbito lateral, no 4. espaço intercostal esquerdo (E.I.E) na linha axilar anterior e as bulhas eram impalpáveis. À ausculta, a 1 o bulha era normofonética, a $2 \%$ bulha desdobrava-se à inspiração e era mais intensa no 2o E.I.E. Teve-se dúvida se havia ou não hiperfonese de $P_{2}$. Havia $S S$ grau II/VI audivel principalmente no $2{ }^{\circ}$ e 3 ? E.I.E. No abdome o lobo direito não foi palpado e o lobo esquerdo estava a $6 \mathrm{~cm}$ do apêndice xifóide. $O$ baço foi palpado debaixo da reborda costal esquerda, com dificuldade e somente à inspiração profunda. Portanto, foi considerado como tendo a forma hépato-intestinal da esquistossomose. Em novembro de 1983 internouse para avaliação clínica. $O$ exame fisico forneceu os mesmos resultados obtidos em 03/02/83. A radiografia de tórax foi normal, o eletrocardiograma mostrou bradicardia sinusal, a ecocardiografia revelou sinais de hipertensão pulmonar: velocidade EF - zero, velocidade $B C-260 \mathrm{~mm} / \mathrm{seg}$., onda "A" - Omm, diâmetro do ventrículo direito/via de saida do ventrículo direito - $25 / 30 \mathrm{~mm}$, cateterismo cardíaco $32 / 12 \mathrm{mmHg}$, média de $20 \mathrm{mmHg}$ na artéria pulmonar. Esofagograma com "questionáveis varizes de esôfago", hematócrito - $42 \%$, hemoglobina $-12,6 \mathrm{~g} \%$, leucócitos 5.700: bas. 2, bast. 5, seg. 48 e linf. 38 .

Após este exame foi confirmado o diagnóstico da forma hépato-intestinal, hipertensão pulmonar é discutivel hipertensão porta.

\section{COMENTÁRIOS}

Em 1972 este paciente era hépato-esplênico e tinha hipertensão pulmonar, com $27,5 \mathrm{mmHg}$ de pressão média no tronco da artéria pulmonar. A hiper- 
Relato de Caso. Emanuel A, Prata A, Bina JC, Guimarães A. Hipertensão pulmonar esquistossomótica persistente em paciente após reversão da forma hépato-esplênica: apresentação de um caso. Revista da Sociedade Brasileira de Medicina Tropical 19:171-172, Jul-Set, 1986

tensão pulmonar existia quando ele apresentava aquela forma clínica. Se não tivéssemos o relato do exame de 1972 poderiamos pensar, errôneamente, que a hipertensão pulmonar se estabeleceu na vigência da forma hépato-intestinal. Nunca vimos hipertensão pulmonar esquistossomótica comprovada se instalar na ausência de hépato-esplenomegalia.

Certamente, pela quimioterapia especifica, houve reversão da forma hépato-esplênica, como sabemos que pode ocorrer ${ }^{3}$. A hipertensão pulmonar pode se beneficiar pela quimioterapia ${ }^{17}$, o que parece ter ocorrido no presente caso, embora ainda persistisse.

Em 1983 o paciente tinha a forma hepatointestinal. A evidência de questionáveis varizes de esôfago faz supor que ele fosse portador de possível hipertensão porta com fibrose de Symmers, conforme está assinalado na literatura ${ }^{13}$. Apesar da dúvida deixada pelo exame clínico quanto à hipertensão pulmonar, o diagnóstico foi firmado em bases hemodinâmicas e ecocardiográficas. Do ponto de vista hemodinâmico consideramos o paciente portador de hipertensão pulmonar por preencher os critérios adotados para tal por Guimarães ${ }^{8}$ e Puigbó ${ }^{14}$, ou seja pressão média na artéria pulmonar igual ou superior a $20 \mathrm{mmHg}$. Com relação ao ecocardiograma, são preenchidos os critérios adotados por Weyman e $\operatorname{cols}^{19} \mathrm{e}$ Shah e cols ${ }^{16}$ : velocidade EF menor que $6 \mathrm{~mm}$ e onda "A" igual ou menor que $2 \mathrm{~mm}$. Além desses parâmetros, as dimensões da cavidade do ventrículo direito e sua via de saída estavam aumentados, traduzindo as repercussões hemodinâmicas da hipertensão pulmonar.

\section{SUMMARY}

The authors report a case of 24 year old man with hepatosplenic schistosomiasis and pulmonary hypertension (mean pulmonary artery arterial pressure $27.5 \mathrm{~mm}$ of mercury). Eleven years after treatment with oxamniquine he had the hepatoinstestinal form of the disease with persistence of the pulmonary hypertension (20 $\mathrm{mm}$ ) as diagnosed by cardiac catheterisation and echocardiography.

Key word: Schistosomaisis. Pulmonary hypertension. Portal hypertension.

\section{REFERÊNCIAS BIBLIOGRÁFICAS}

1. Azmy BS. Pulmonary arteriosclerosis of bilharzial nature. Journal of the Egyptian Medical Association 15: 87-90, 1932.

2. Barbato ECD. Pneumopatia e cor-pulmonale crônico esquistossomótico. São Paulo. Faculdade de Medicina da Universidade de São Paulo. Tese, 1952.
3. Bina JC. Influência da terapêutica especifica na evolução da esquistossomose mansoni. Tese. Salvador, Faculdade de Medicina Universidade Federal da Bahia, 1977.

4. Cavalcanti IL, Tompson G, Souza N, Barbosa FS. Pulmonary hypertension in schistosomiasis. British Heart Journal 24: 363-371, 1962.

5. Coelho EM, Duarte CS. The syndrome of portopulmonary schistosomiasis. American Journal of Medicine 43: 944-950, 1967.

6. Gelfand $M$. Cor-pulmonale and cardio-pulmonary schistosomiasis. Transactions of the Royal Society of Tropical Medicine and Hygiene 51: 533-540, 1957.

7. Girgis B. Pulmonary heart disease due to bilharzia. The bilharzia cor-pulmonale. American Heart Journal 43: 606-614, 1952.

8. Guimarães AC. Situação atual do conhecimento sobre o envolvimento cardio-pulmonar na esquistossomose mansonica. Arquivos Brasileiros de Cardiologia 38: 301-309, 1982.

9. Ibrahim N, Girgis B. Bilharzial cor-pulmonale. A clinicopathological report of 50 cases. Journal of Tropical Medicine and Hygiene 63: 55-58, 1960.

10. Kenawy MR. The syndrome of cardio-pulmonary schistosomiasis. American Heart Journal 39: 678-696, 1950.

11. Marchand EJ, Marcial-Rojas RA, Rodriguez R, Polanco $G$, Diaz-Rivera RS. The pulmonary obstruction syndrome in Schistosoma mansoni pulmonary endarteritis. Archives of Internal Medicine 100: 965-980, 1957.

12. Meira JA. Estudo clínico das formas pulmonares da esquistossomose mansônica. Arquivos de Cirurgia Clinica e Experimental 6: 3-136, 1942.

13. Prata A, Andrade Z. Fibrose-hepática de Symmers sem esplenomegalia. Hospital 63: 617-623, 1963.

14. Puigbó JJ. Valores normais. Pression. Flujo. Resistencia. Trabajo cardiaco. Contenido de oxigenio. In: Puigbó, Blanco, Machado e Giordano (eds). Bases hemodinamicas de la clínica cardiovascular. Caracas, Inprenta Universitaria, 1966.

15. Santiago JM, Ratton JLA. Formas clinicas da esquistossomose pulmonar crônica. Subsidios para sua classificação. Revista da Associação Médica de Minas Gerais 19: 62-81, 1968.

16. Shah A, Schwartz H, Closs RN. Unusual echocardiographic findings in primary pulmonary hypertension. Archives of Internal Medicine 143: 820-822, 1983.

17. Silva AE. Aspectos clínico-epidemiológico e evolutivo da hipertensão pulmonar esquistossomótica. Tese. Núcleo de Medicina Tropical e Nutrição da Universidade de Brasilia, Brasilia, 1984.

18. Silveira J. Esquistossomose do pulmão e tuberculose pulmonar. Imprensa Regina, Bahia, 1941.

19. Weyman AE, Dillon JC, Feigenbaum H, Chang S. Echocardiographic patterns of pulmonic valve motion with pulmonary hypertension. Circulation 50: 905-910, 1974. 